\title{
The effect of dietary and/or cosmetic argan oil on postmenopausal skin elasticity
}

\author{
This article was published in the following Dove Press journal: \\ Clinical Interventions in Aging \\ 30 January 2015 \\ Number of times this article has been viewed
}

\author{
Kenza Qiraouani Boucetta' \\ Zoubida Charrouf ${ }^{2}$ \\ Hassan Aguenaou ${ }^{3}$ \\ Abdelfattah Derouiche ${ }^{4}$ \\ Yahya Bensouda' \\ 'Research Team on Formulation \\ and Biopharmacy, Research Center \\ for Drug, Faculty of Medicine and \\ Pharmacy, Mohammed V University, \\ Rabat, Morocco; ${ }^{2}$ Faculty of Sciences, \\ Mohammed V University, Rabat, \\ Morocco; ${ }^{3}$ Mixed Unit of Research in \\ Nutrition, ITU / CNESTEN, Ibn Tofail \\ University, Kenitra, Morocco; ${ }^{4}$ Faculty \\ of Sciences, Hassan II University, \\ Casablanca, Morocco
}

Background: During menopause, the decrease of estrogenic secretion induces the disruption of skin functioning, thus causing the decline in skin elasticity characteristic of skin aging. The purpose of this study was to evaluate in postmenopausal women the effect of daily consumption and/or application of argan oil on skin elasticity.

Materials and methods: Sixty postmenopausal women consumed butter during the stabilization period and were randomly divided into two groups for the intervention period: the treatment group of 30 participants received dietary argan oil, the control group of 30 participants received olive oil, and both groups applied cosmetic argan oil in the left volar forearm during a 60-day period. Assessments of skin elasticity parameters, ie, the three R-parameters (R2 or grosselasticity of the skin, R5 or net elasticity of the skin, and R7 or biological elasticity), and the resonance running time (RRT) at both volar forearms of the two groups were performed during three visits: before starting oils consumption and application, after 30 days of oils consumption and application, and after 60 days of oils consumption and application.

Results: The consumption of argan oil led to a significant increase of gross-elasticity of the skin (R2) $(P<0.001)$, net elasticity of the skin (R5) $(P<0.001)$, biological elasticity (R7) $(P<0.001)$, and a significant decrease of RRT $(P=0.002)$. The application of argan oil led to a significant increase of gross-elasticity of the skin (R2) $(P<0.001)$, net elasticity of the skin (R5) $(P<0.001)$, biological elasticity (R7) $(P=0.001)$, and a significant decrease of RRT $(P<0.001)$.

Conclusion: Our findings suggest that the daily consumption and/or topical application of argan oil have an anti-aging effect on the skin demonstrated by the improvement of skin elasticity, characterized by an increase of R-parameters (R2, R5, and R7) and a decrease of RRT.

Keywords: postmenopausal women, argan oil, skin elasticity

\section{Introduction}

The skin is stratified into two essential superposed layers: the outermost layer or epidermis and the deeper layer or dermis.

The dermis provides a solid support for the epidermis. It is also its feeder layer. It consists mainly of fibroblasts, cells responsible for collagen and elastic fibers synthesis that enter into the extracellular matrix composition. ${ }^{1}$

Elastic fibers are closely linked and interwoven with the collagen fibrils so that they can recoil after transient stretching, preventing overstretching.

The presence of collagen and elastic fibers or the major (97.5\%) and the minor $(2.5 \%)$ compounds of dermis, respectively, promote the maintenance of resilience and elastic appearance of the skin. ${ }^{2}$

The decrease of estrogenic secretion observed during menopause negatively affects the skin elasticity by a decrease of dermal fibroblasts collagen ${ }^{3}$ and elastic
Correspondence: Yahya Bensouda Research Team on Formulation and Biopharmacy, Faculty of Medicine and Pharmacy of Rabat University Mohammed $\checkmark$ at Souissi, PO Box 6203, Morocco Tel +2I266I3329 9I

Fax +2I 253777370 I

Email y.bensouda@um5s.net.ma 
fibers, ${ }^{4}$ characteristic of skin aging ${ }^{5}$ this is the main reason we chose healthy postmenopausal women as the test population for this study.

Treatment with estrogen reverses the changes of collagen and elastic fibers, ${ }^{6,7}$ which favor the improvement of skin elasticity in postmenopausal women. ${ }^{8}$ But the long-term use of estrogen in the form of hormone replacement therapy triggers many pathophysiological events. ${ }^{9}$

The consumption of dietary argan oil has proved to have a positive effect in the prevention of certain diseases (high cholesterol, ${ }^{10}$ heart disease,${ }^{11}$ diabetes, ${ }^{12}$ etc) that are known to have a high incidence in postmenopausal women ${ }^{13}$ and which may thus be beneficial in treating reduced skin elasticity during menopause.

Although argan oil has a long history in traditional cosmetic use, to maintain the health of skin, ${ }^{14}$ its effect on skin elasticity has never been scientifically reported. Therefore, we have attempted to elucidate the effect of argan oil on skin elasticity.

The purpose of this study was to evaluate in postmenopausal women the effect of daily consumption and topical application of argan oil on skin elasticity, as measured by the skin's biomechanical properties that are represented by R-parameters (R2 or gross-elasticity of the skin, R5 or net elasticity of the skin, and R7 or biological elasticity), and resonance running time (RRT) using noninvasive biophysical methods.

\section{Materials and methods Participants}

One hundred postmenopausal women were recruited in our study; 60 of them met the following criteria:

Inclusion criteria: menopause confirmed by dosages of estradiol and follicle-stimulating hormone (estradiol $<30 \mathrm{pg} / \mathrm{mL}$ and follicle-stimulating hormone $>40 \mathrm{IU} / \mathrm{L}$ ) and absence of skin diseases.

Exclusion criteria: taking a hormone replacement therapy, and use of moisturizing and anti-aging products and dietary supplements.

The 60 postmenopausal women selected gave their informed consent and the study was approved by the Ethical Committee for Biomedical Research of the Faculty of Medicine and Pharmacy, University Mohammed V Rabat, Morocco.

\section{Products}

The following products were used in this study: butter, olive oil of the Moroccan Picholine, and dietary and cosmetic argan oil provided by the cooperative Targante with the ECOCERT label.

\section{Study protocol}

In this study, there were two diet periods (Figure 1). In diet period 1 (baseline diet), all of the participants consumed $25 \mathrm{~g} /$ day of the same brand of butter for 2 weeks to stabilize the lipid profile. ${ }^{15}$ In diet period 2, the 60 participants were randomly assigned to the two following diet groups using the "Data - Select cases - Random sample of cases" procedure in SPSS (version 10, IBM Corporation, Armonk, NY, USA): one group of 30 participants, in which $25 \mathrm{~g} /$ day butter was substituted by $25 \mathrm{~mL} /$ day of argan oil, and the second group of 30 participants consuming $25 \mathrm{~mL}$ /day of olive oil as a control during a 60 -day period. In parallel, the two groups were asked to apply every night about $240 \mathrm{mg}$ $\left(2 \mathrm{mg} / \mathrm{cm}^{2}\right)$ of cosmetic argan oil, corresponding to 10 drops, on the left volar forearm (LF) and were forbidden to use any other skin care or cosmetic products on their volar forearms throughout the study.

Cosmetic argan oil and alimentary argan and olive oil bottles were delivered to participants every 30 days by the research staff, in order to meet the participants and ensure their adherence to the study. The residual alimentary and cosmetic oil bottles were collected at the time of the next delivery in order to check the adherence of the participants to the allocated treatment. Olive oil was deliberately chosen as the oil of the control group to ensure adherence to the study of women not receiving argan oil, as olive oil is also considered an oil with noble nutritional virtues and widely known for its beneficial health effects.

In the two groups, assessments of skin elasticity, ie, the R-parameters (R2, R5, and R7), and the RRT at the right volar forearm (RF) and the LF were performed in the morning during three visits: before starting oil consumption and application (D0), after 30 days of oil consumption and application (D30), and after 60 days of oil consumption and application (D60).

\section{Bioengineering methods}

The measurements of skin elasticity were performed with a Cutometer SEM 575 ${ }^{\circledR}$ and a Reviscometer RVM 600 ${ }^{\circledR}$, which were mounted on a Multi Probe Adapter ${ }^{\circledR}$ MPA5 (Courage and Khazaka Electronic GmbH, Cologne, Germany).

The Cutometer SEM 575 with a $2 \mathrm{~mm}$ diameter probe is an electronic instrument that assesses biomechanical properties of the skin based on the suction and elongation measuring principle. The time/strain mode (mode 1) was 
The phase of recruitment and

stabilization of PMW volunteers

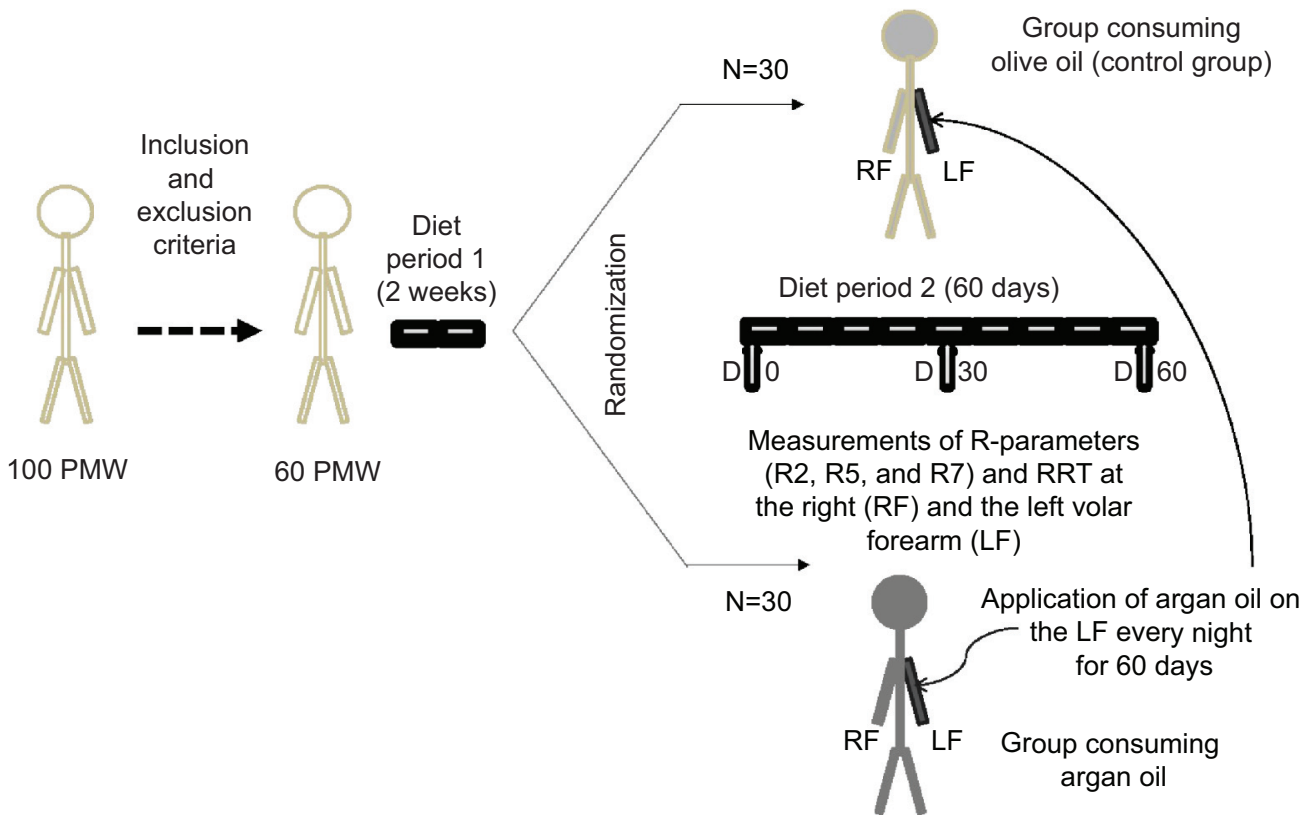

Figure I Overall protocol of the study.

Abbreviations: D0, before starting oil consumption and application; D30, after 30 days of oil consumption and application; D60, after 60 days of oil consumption and application; LF, left volar forearm; PMW, postmenopausal women; R2, gross-elasticity of the skin; R5, net elasticity of the skin; R7, biological elasticity; RF, right volar forearm; RRT, resonance running time.

used with ten consecutive cycles of a 2-second application of a constant negative pressure of $450 \mathrm{mbar}$, followed by a 2-second relaxation period.

A typical skin deformation curve is illustrated in Figure 2 and the following parameters were analyzed: (R2) Ua (the ability of redeformation of skin)/Uf (final distension [skin distensibility]), gross-elasticity of the skin, including viscous deformation; ${ }^{16}$ (R5) Ur (immediate retraction)/Ue (immediate

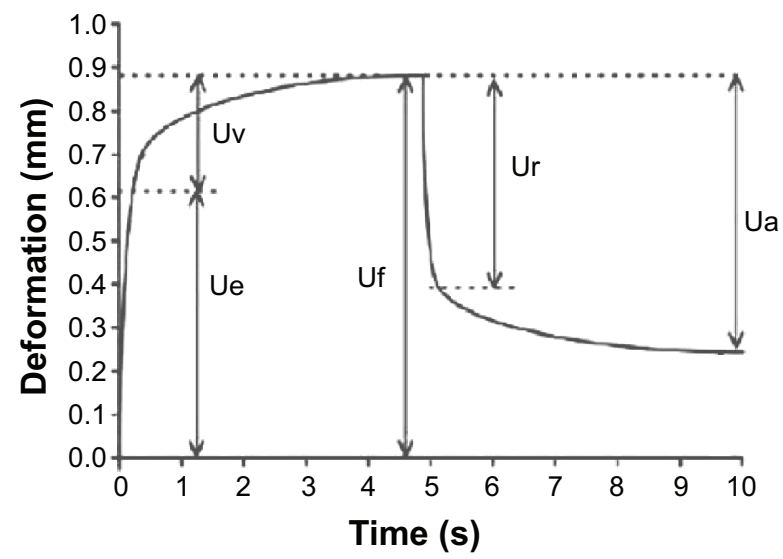

Figure 2 Skin deformation curve obtained with Cutometer.

Abbreviations: $U a$, the ability of redeformation of skin; Ue, immediate distension; Uf, final distension (skin distensibility); Ur, immediate retraction; Uv, delayed distension; s, seconds. distension), net elasticity of the skin without viscous deformation; ${ }^{16}$ (R7) Ur/Uf, biological elasticity, ie the ratio of immediate retraction to final distension. ${ }^{16}$ The Reviscometer RVM 600 also allows for the evaluation of the mechanical properties of skin by measuring the propagation time of a shear wave between two sensors placed on the skin surface: one is transmitting an acoustical shockwave, and the other is the receiver. The time the wave needs to propagate from transmitter to recipient is the measured parameter that is defined as RRT. It has been shown that RRT is inversely related to skin elasticity. ${ }^{17}$ The longer the time the waves need to propagate through the material, the higher the measuring value and the less elastic the material. We measured RRT according to manufacturer instructions.

\section{Statistical analysis}

All values are expressed as mean \pm standard deviation (SD). R-parameters (R2, R5, and R7), RRT values, and other variables were normally distributed as assessed by the Kolmogorov-Smirnov test.

The parametric test, analysis of variance (ANOVA) for repeated measures, was applied for comparisons of the three paired means ( $\pm \mathrm{SD}$ ) of R2, R5, R7, and RRT (taken at D0, D30, and D60) of the two volar forearms (RF and LF) of each 
Table I Baseline demographic and clinical characteristics of the argan and olive groups

\begin{tabular}{lll}
\hline Item & Olive group (control) & Argan group \\
\hline $\mathrm{n}$ & 30 & 30 \\
Age, years & $55.13 \pm 6.45$ & $55.84 \pm 5.9 \mathrm{I}$ \\
Body weight, $\mathrm{kg}$ & $70.02 \pm 12.27$ & $69.94 \pm \mathrm{II} .85$ \\
Height, $\mathrm{m}$ & $1.58 \pm 0.06$ & $1.57 \pm 0.05$ \\
$\mathrm{TC}, \mathrm{g} / \mathrm{L}$ & $2.10 \pm 0.33$ & $2.13 \pm 0.35$ \\
$\mathrm{LDL}-\mathrm{C}, \mathrm{g} / \mathrm{L}$ & $1.30 \pm 0.3 \mathrm{I}$ & $\mathrm{I} .34 \pm 0.3 \mathrm{I}$ \\
$\mathrm{HDL}-\mathrm{C}, \mathrm{g} / \mathrm{L}$ & $0.58 \pm 0.12$ & $0.58 \pm 0.15$ \\
$\mathrm{TG}, \mathrm{g} / \mathrm{L}$ & $1.10 \pm 0.56$ & $1.09 \pm 0.5 \mathrm{I}$ \\
\hline
\end{tabular}

Notes: All values are presented as unadjusted mean \pm standard deviation. Abbreviations: LDL-C, low-density lipoprotein cholesterol; HDL-C, high-density lipoprotein cholesterol; TC, total cholesterol; TG, triglycerides.

group (argan and olive). When the ANOVA test for repeated measures was significant, the Bonferroni test was applied to perform post hoc pairwise multiple comparisons within the three paired means ( \pm SD) of R2, R5, R7, and RRT.

The Student's $t$-test for independent samples was used to compare the mean of the R2, R5, R7, and RRT between the two forearms of each group at the beginning of the study (at D0) to prove the homogeneity of the results and at the end of the study (at D60) to support the results of the ANOVA for repeated measures.

Differences were considered to be statistically significant at $P<0.05$ and the statistical software SPSS version 10.0 was used.

\section{Results}

Baseline demographic and clinical characteristics of participants treated with argan and olive oil in both groups are shown in Table 1.

\section{Results of the untreated forearm or RF}

In the group consuming argan oil, the paired means $( \pm \mathrm{SD})$ of R-parameters (R2, R5, and R7) and RRT values as a function of time (D0, D30, and D60) showed a statistically significant difference (at the $P<0.05$ level) between at least two means ( \pm SDs) of R-parameters (Figure 3A, Figure 4A,
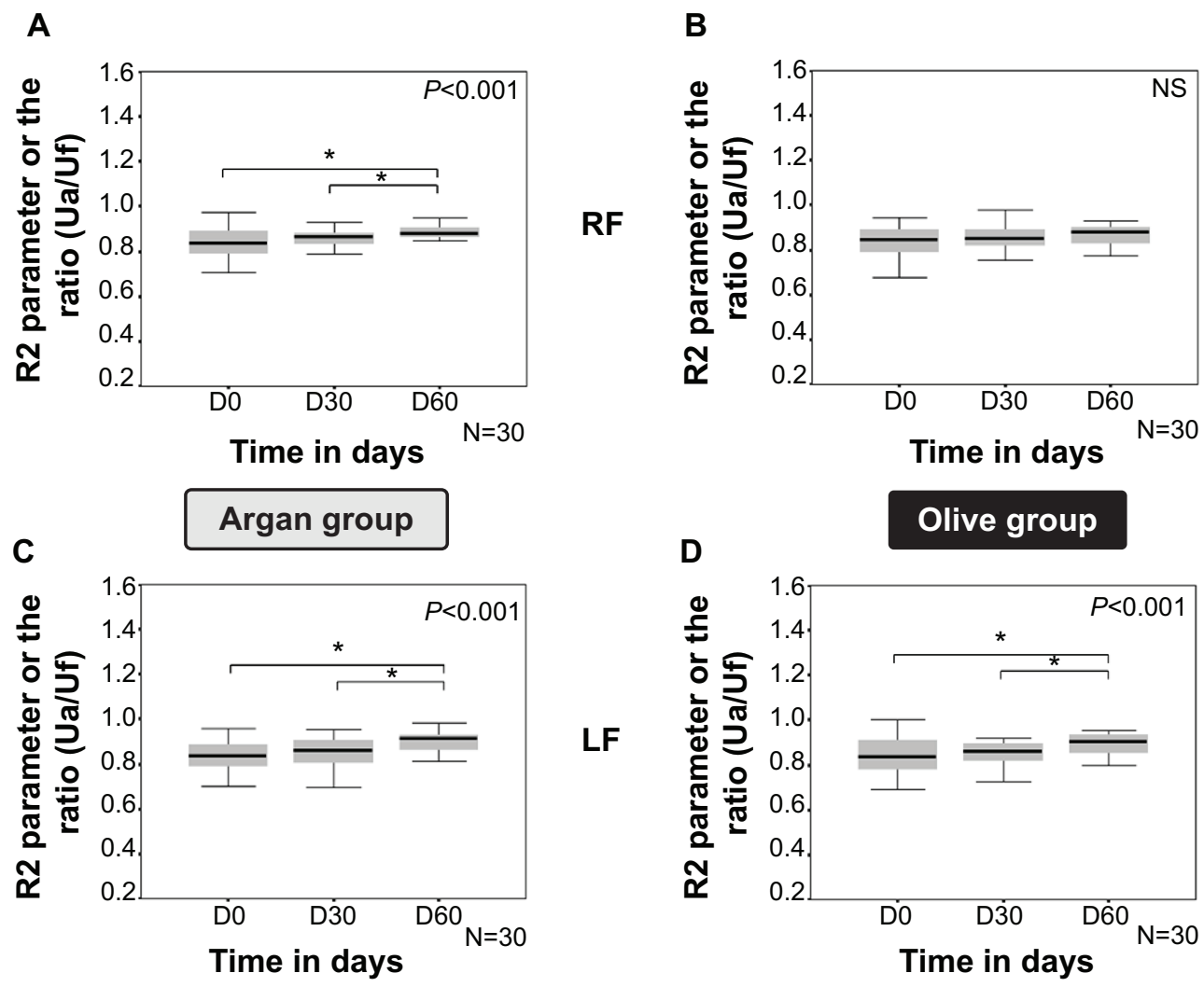

Figure 3 Volar forearm R2 (gross-elasticity of the skin) evolution.

Notes: Evolution after oils consumption and/or argan oil application at (A) the RF in the group consuming argan oil, (B) the RF in the group consuming olive oil, (C) the LF in the group consuming argan oil, and (D) the LF in the group consuming olive oil. The difference between the three paired means ( \pm SD) of R2 (taken at D0, D30, and D60) of the two volar forearms (RF and LF) of each group (argan and olive) was analyzed using the ANOVA test for repeated measures followed by the Bonferroni post hoc test (if the ANOVA test for repeated measures was statistically significant). $P<0.05$ was considered statistically significant (for the results of the ANOVA test for repeated measures). NS: ANOVA test for repeated measures showed no statistically significant difference $(P>0.05)$. $* P<0.05$ was considered statistically significant (for the results of the Bonferroni post hoc test).

Abbreviations: ANOVA, analysis of variance; D0, before starting oil consumption and application; D30, after 30 days of oil consumption and application; D60, after 60 days of oil consumption and application; LF, left volar forearm; NS, no significant difference; RF, right volar forearm; SD, standard deviation; Ua/Uf, gross-elasticity of the skin, including viscous deformation. 
A
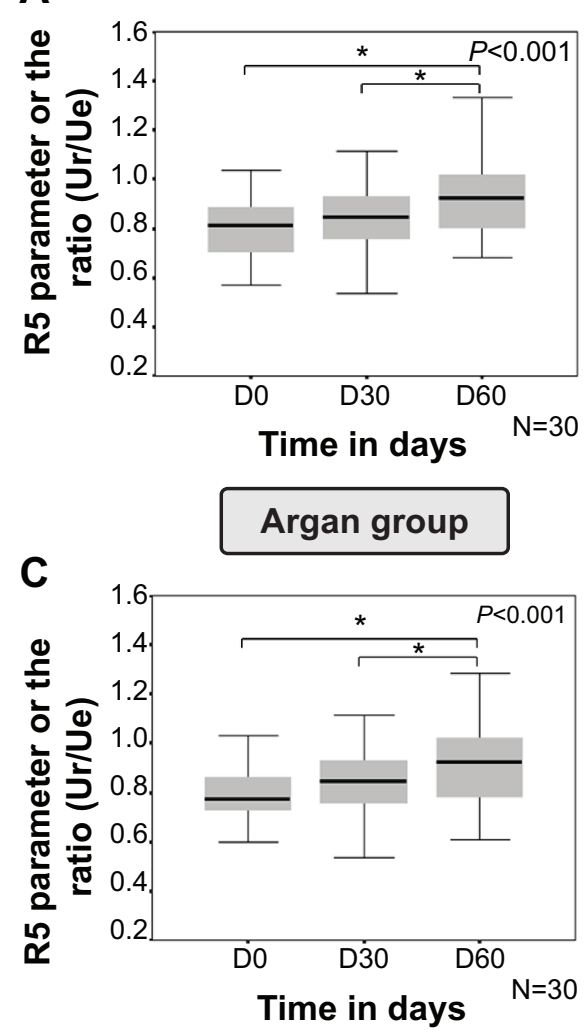

B

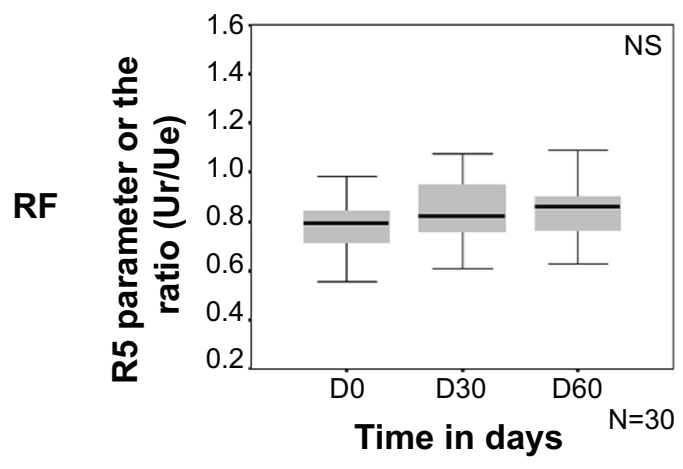

Olive group

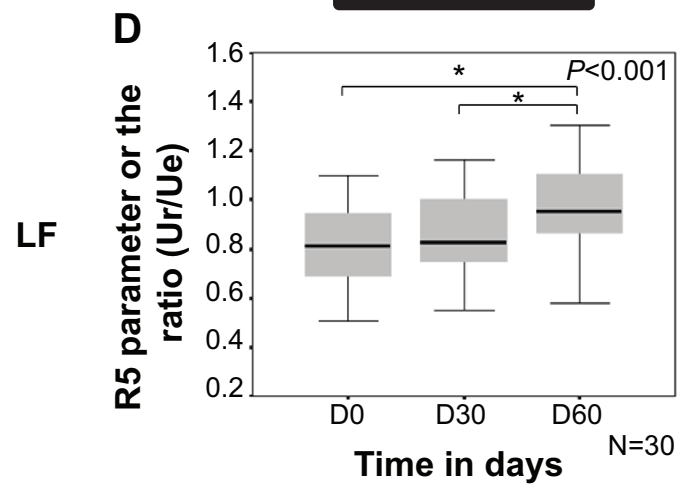

Figure 4 Volar forearm R5 (net elasticity of the skin without viscous deformation) evolution.

Notes: Evolution after oils consumption and/or argan oil application at (A) the RF in the group consuming argan oil, (B) the RF in the group consuming olive oil, (C) the LF in the group consuming argan oil, and (D) the LF in the group consuming olive oil. The difference between the three paired means ( \pm SD) of R5 (taken at D0, D30, and D60) of the two volar forearms (RF and LF) of each group (argan and olive) was analyzed using the ANOVA test for repeated measures followed by the Bonferroni post hoc test (if the ANOVA test for repeated measures was statistically significant). $P<0.05$ was considered statistically significant (for the results of the ANOVA test for repeated measures). NS: the ANOVA test for repeated measures showed no statistically significant difference $(P>0.05)$. $* P<0.05$ was considered statistically significant (for the results of the Bonferroni post hoc test).

Abbreviations: ANOVA, analysis of variance; D0, before starting oil consumption and application; D30, after 30 days of oil consumption and application; D60, after 60 days of oil consumption and application; LF, left volar forearm; NS, no significant difference; RF, right volar forearm; SD, standard deviation; Ur/Ue, net elasticity of the skin without viscous deformation.

and Figure 5A) and RRT (Figure 6A) with a level of significance of $P<0.001$ and $P=0.002$, respectively. This statistically significant difference existed between the D0 and the D60 mean $( \pm \mathrm{SD})$ for R2 $(P=0.001$; Figure $3 \mathrm{~A})$, R5 $(P<0.001$; Figure 4A), R7 $(P<0.001$; Figure 5A), and RRT $(P=0.002$; Figure 6A) and between the D30 and the D60 mean $( \pm \mathrm{SD})$ for $\mathrm{R} 2(P=0.019$; Figure $3 \mathrm{~A})$, R5 ( $P=0.006$; Figure 4A), R7 ( $P=0.011$; Figure 5A), and RRT ( $P=0.001$; Figure $6 \mathrm{~A})$. No statistically significant difference was observed between at least two means ( \pm SDs) of R-parameters ( $P>0.05$; Figure 3B, Figure 4B, and Figure 5B) and RRT values $(P>0.05$; Figure $6 \mathrm{~B})$ in the group consuming olive oil.

Between the group consuming argan oil and that consuming olive oil, there was no statistically significant difference in the unpaired means $( \pm \mathrm{SD})$ of the means $( \pm \mathrm{SD})$ of R-parameters $(P>0.05)$ and RRT $(P>0.05)$ at baseline (D0), but there was a statistically significant difference after the 60-day period (D60) in favor of the group consuming argan oil for the R2 ( $P=0.036), \mathrm{R} 5(P=0.036), \mathrm{R} 7(P=0.012)$, and RRT values $(P=0.006)$ (Table 2$)$.

\section{Results of the treated forearm or LF}

The paired means $( \pm \mathrm{SD})$ of R-parameters (R2, R5, and R7) and RRT values as a function of time (D0, D30, and D60) showed a statistically significant difference between at least two means ( \pm SDs) of R2, R5, R7, and RRT with a level of significance of $P<0.001, P<0.001, P=0.001$ (Figure 3C, Figure 4C, and Figure 5C), and $P<0.001$ (Figure 6C), respectively, in the group consuming argan oil, and with a level of significance of $P<0.001$ (Figure 3D, Figure 4D, Figure 5D) and $P=0.008$ (Figure 6D), respectively, in the group consuming olive oil. This statistically significant difference existed between the D0 and the D60 means ( \pm SD) for R2 ( $P<0.001$ in the two groups; Figure 3C, D), R5 ( $P=0.006$ in the group consuming argan oil and $P<0.001$ in 
A
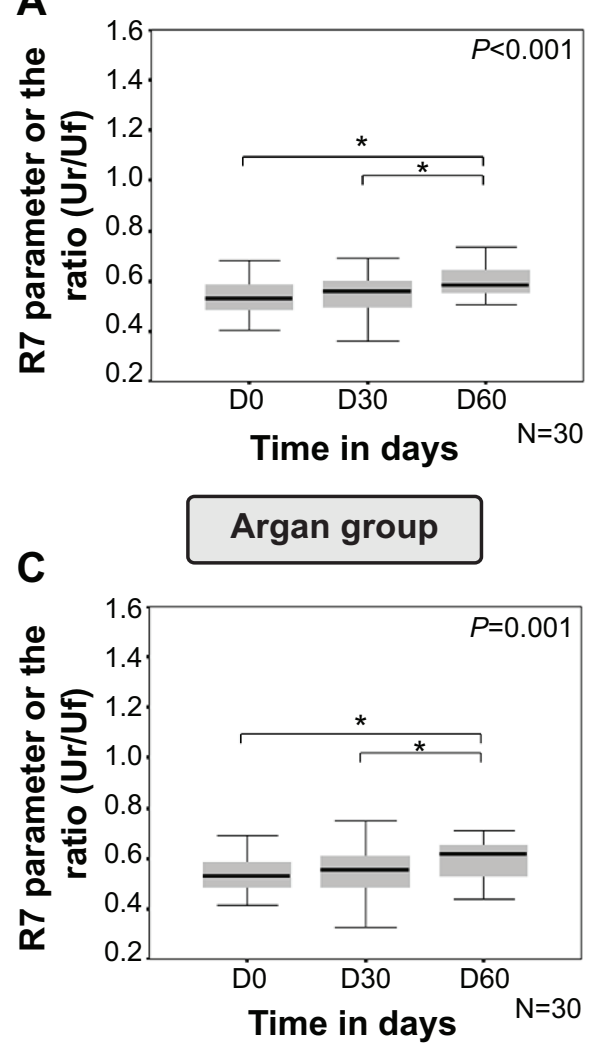

B

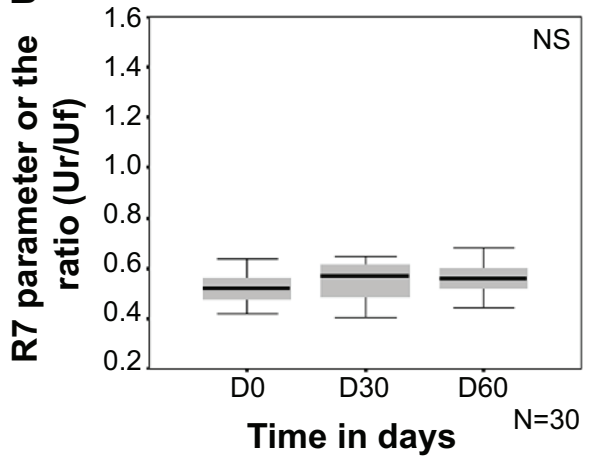

Olive group

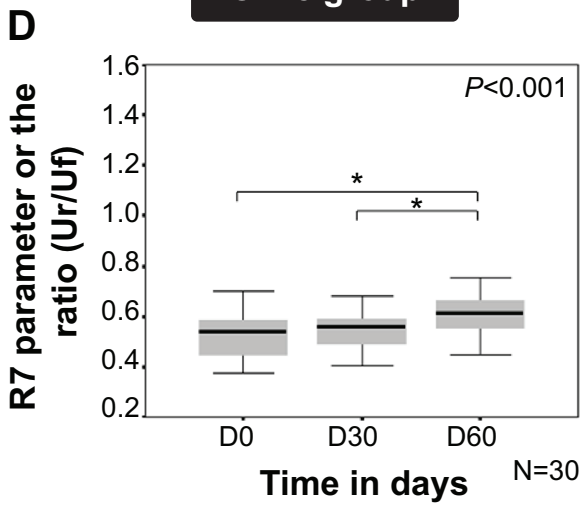

Figure 5 Volar forearm R7 (biological elasticity) evolution.

Notes: Evolution after oils consumption and/or argan oil application at (A) the RF in the group consuming argan oil, (B) the RF in the group consuming olive oil, (C) the LF in the group consuming argan oil, and (D) the LF in the group consuming olive oil. The difference between the three paired means ( \pm SD) of R7 (taken at D0, D30, and D60) of the two volar forearms (RF and LF) of each group (argan and olive) was analyzed using the ANOVA test for repeated measures followed by the Bonferroni post hoc test (if the ANOVA test for repeated measures was statistically significant). $P<0.05$ was considered statistically significant (for the results of the ANOVA test for repeated measures). NS: the ANOVA test for repeated measures showed no statistically significant difference $(P>0.05)$. $* P<0.05$ was considered statistically significant (for the results of the Bonferroni post hoc test).

Abbreviations: ANOVA, analysis of variance; D0, before starting oil consumption and application; D30, after 30 days of oil consumption and application; D60, after 60 days of oil consumption and application; LF, left volar forearm; NS, no significant difference; RF, right volar forearm; SD, standard deviation; Ur/Uf, biological elasticity, ie, the ratio of immediate retraction to final distension.

the group consuming olive oil; Figure 4C, D), R7 ( $P<0.001$ in the two groups; Figure 5C, D), and RRT $(P<0.001$ in the group consuming argan oil and $P=0.022$ in the group consuming olive oil; Figure $6 \mathrm{C}, \mathrm{D})$, and between the D30 and the D60 means $( \pm \mathrm{SD})$ for $\mathrm{R} 2(P=0.004$ in the group consuming argan oil and $P=0.002$ in the group consuming olive oil; Figure 3C, D), R5 ( $P=0.007$ in the group consuming argan oil and $P=0.001$ in the group consuming olive oil; Figure $4 \mathrm{C}, \mathrm{D}), \mathrm{R} 7$ ( $P=0.010$ in the group consuming argan oil and $P<0.001$ in the group consuming olive oil; Figure $5 \mathrm{C}, \mathrm{D})$, and RRT ( $P=0.001$ in the group consuming argan oil and $P=0.022$ in the group consuming olive oil; Figure 6C, D).

Between the group consuming argan oil and that consuming olive oil, there was no statistically significant difference in the unpaired means $( \pm \mathrm{SD})$ of R-parameters $(P>0.05)$ and RRT values $(P>0.05)$ at baseline (D0) and after the 60-day period (D60) (Table 2).

\section{Comparison of topical-argan-oil-treated and -untreated forearms, or LF and RF, in the two groups}

Between the RF and the LF in the two groups (argan and olive), there was no statistically significant difference in the unpaired means $( \pm \mathrm{SD})$ of R-parameters $(P>0.05)$ and the RRT $(P>0.05)$ at baseline (D0), but there was a statistically significant difference after the 60-day period (D60) in favor of the LF in the group consuming olive oil with $P=0.023$, $P=0.004, P=0.005$, and $P=0.022$ for the R2, R5, R7, and RRT values, respectively (Table 3 ).

\section{Discussion}

In this study we have demonstrated that the daily consumption and topical application of argan oil improves skin elasticity by increasing the R-parameters (R2, R5, and R7) and decreasing the RRT in postmenopausal women after a period of 60 days. 

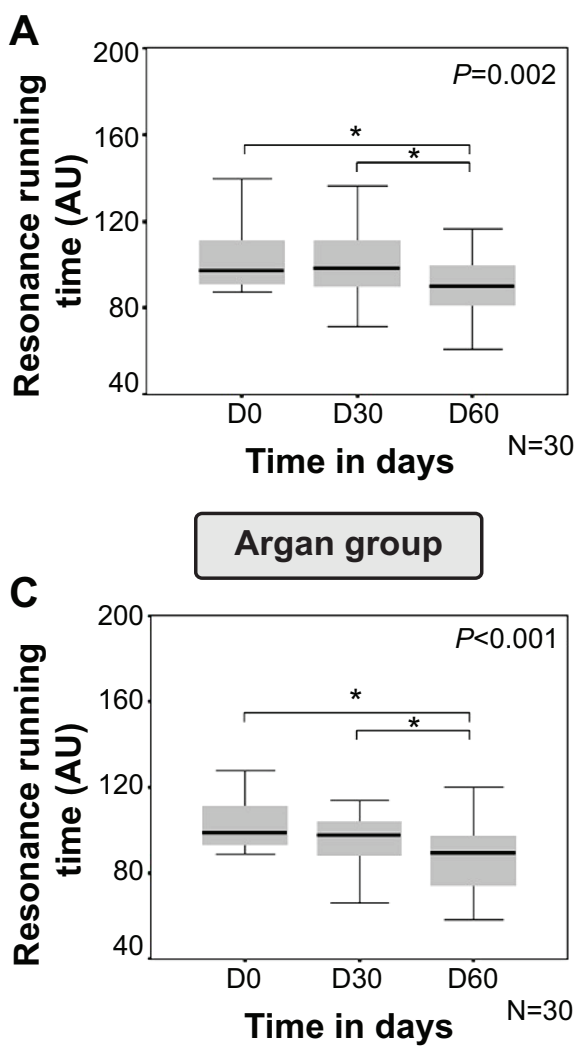

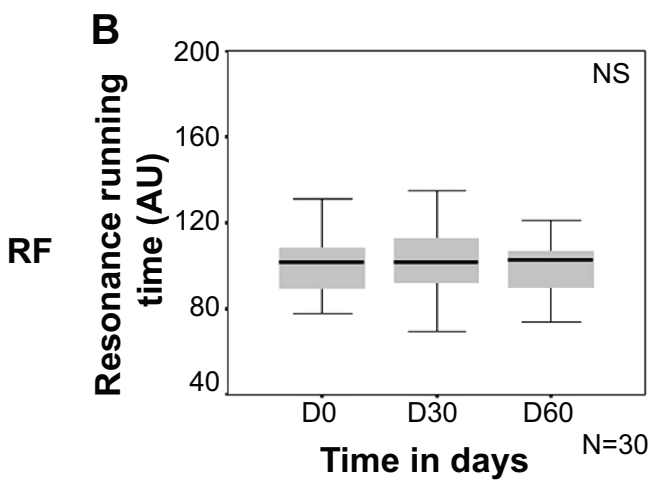

Olive group

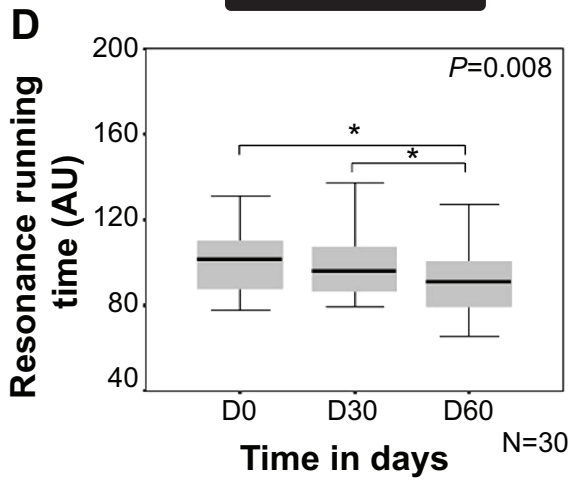

Figure 6 Volar forearm RRT evolution.

Notes: Evolution after oils consumption and/or argan oil application at (A) the RF in the group consuming argan oil, (B) the RF in the group consuming olive oil, (C) the LF in the group consuming argan oil, (D) the LF in the group consuming olive oil. The difference between the three paired means ( \pm SD) of RRT (taken at D0, D30, and D60) of the two volar forearms (RF and LF) of each group (argan and olive) was analyzed using the ANOVA test for repeated measures followed by the Bonferroni post hoc test (if the ANOVA test for repeated measures was statistically significant). $P<0.05$ was considered statistically significant (for the results of the ANOVA test for repeated measures). NS: the ANOVA test for repeated measures showed no statistically significant difference $(P>0.05)$. $* P<0.05$ was considered statistically significant (for the results of the Bonferroni post hoc test).

Abbreviations: ANOVA, analysis of variance; AU, arbitrary units; D0, before starting oil consumption and application; D30, after 30 days of oil consumption and application; D60, after 60 days of oil consumption and application; LF, left volar forearm; NS, no significant difference; RF, right volar forearm; RRT, resonance running time; SD, standard deviation.

Table 2 The intergroup comparison of R-parameters (R2, R5, and R7) and resonance running time of the volar forearm between the two groups before (D0) and after 60 days (D60) of oils consumption and/or argan oil application

\begin{tabular}{|c|c|c|c|c|c|}
\hline & & & $\begin{array}{l}\text { Olive group } \\
\mathbf{N}=30\end{array}$ & $\begin{array}{l}\text { Argan group } \\
\mathbf{N}=30\end{array}$ & $P$-value \\
\hline $\mathrm{R} 2$ parameter or the ratio & RF & D0 & $0.836 \pm 0.067$ & $0.838 \pm 0.077$ & $>0.05$ \\
\hline \multirow[t]{3}{*}{$(\mathrm{Ua} / \mathrm{Uf})$} & & D60 & $0.855 \pm 0.072$ & $0.886 \pm 0.029$ & 0.036 \\
\hline & LF & D0 & $0.836 \pm 0.083$ & $0.837 \pm 0.063$ & $>0.05$ \\
\hline & & D60 & $0.893 \pm 0.049$ & $0.895 \pm 0.048$ & $>0.05$ \\
\hline R5 parameter or the ratio & RF & D0 & $0.793 \pm 0.12$ & $0.798 \pm 0.12$ & $>0.05$ \\
\hline \multirow[t]{3}{*}{$(\mathrm{Ur} / \mathrm{Ue})$} & & D60 & $0.850 \pm 0.144$ & $0.940 \pm 0.188$ & 0.036 \\
\hline & LF & D0 & $0.797 \pm 0.16$ & $0.80 I \pm 0.103$ & $>0.05$ \\
\hline & & D60 & $0.993 \pm 0.218$ & $0.937 \pm 0.192$ & $>0.05$ \\
\hline R7 parameter or the ratio & RF & D0 & $0.525 \pm 0.058$ & $0.53 I \pm 0.086$ & $>0.05$ \\
\hline \multirow[t]{3}{*}{$(U r / U f)$} & & D60 & $0.55 I \pm 0.074$ & $0.594 \pm 0.059$ & 0.012 \\
\hline & LF & Do & $0.525 \pm 0.085$ & $0.533 \pm 0.064$ & $>0.05$ \\
\hline & & D60 & $0.607 \pm 0.075$ & $0.595 \pm 0.079$ & $>0.05$ \\
\hline Resonance running time & RF & D0 & $102.1 \pm 15.26$ & $103.70 \pm 17.24$ & $>0.05$ \\
\hline \multirow[t]{3}{*}{$(A U)$} & & D60 & $99.48 \pm 11.98$ & $90.23 \pm 12.95$ & 0.006 \\
\hline & LF & D0 & $101.59 \pm 15.78$ & $104.76 \pm 16.73$ & $>0.05$ \\
\hline & & D60 & $91.29 \pm 14.82$ & $87.09 \pm 14.33$ & $>0.05$ \\
\hline
\end{tabular}

Notes: All values are presented as mean \pm standard deviation. Mean group comparisons were conducted using Student's $t$-test for independent samples. $P<0.05$ was considered statistically significant.

Abbreviations: AU, arbitrary units; LF, left volar forearm; R2, Ua/Uf, gross-elasticity of the skin, including viscous deformation; R5, Ur/Ue, net elasticity of the skin without viscous deformation; R7, Ur/Uf, biological elasticity, ie, the ratio of immediate retraction to final distension; RF, right volar forearm. 
Table 3 The intergroup comparison of R-parameters (R2, R5, and R7) and resonance running time between the two volar forearms (RF and LF) before (D0) and after 60 days (D60) of oils consumption and/or argan oil application in the two groups

\begin{tabular}{|c|c|c|c|c|c|}
\hline & & & $\mathbf{R F}$ & LF & $P$-value \\
\hline $\mathrm{R} 2$ parameter or the ratio & Argan group & D0 & $0.838 \pm 0.077$ & $0.837 \pm 0.063$ & $>0.05$ \\
\hline \multirow[t]{3}{*}{$(\mathrm{Ua} / \mathrm{Uf})$} & $\mathrm{N}=30$ & D60 & $0.886 \pm 0.029$ & $0.895 \pm 0.048$ & $>0.05$ \\
\hline & Olive group & D0 & $0.836 \pm 0.067$ & $0.836 \pm 0.083$ & $>0.05$ \\
\hline & $\mathrm{N}=30$ & D60 & $0.855 \pm 0.072$ & $0.893 \pm 0.049$ & 0.023 \\
\hline R5 parameter or the ratio & Argan group & D0 & $0.798 \pm 0.12$ & $0.80 I \pm 0.103$ & $>0.05$ \\
\hline \multirow[t]{3}{*}{$(\mathrm{Ur} / \mathrm{Ue})$} & $N=30$ & D60 & $0.940 \pm 0.188$ & $0.937 \pm 0.192$ & $>0.05$ \\
\hline & Olive group & D0 & $0.793 \pm 0.12$ & $0.797 \pm 0.16$ & $>0.05$ \\
\hline & $\mathrm{N}=30$ & D60 & $0.850 \pm 0.144$ & $0.993 \pm 0.218$ & 0.004 \\
\hline R7 parameter or the ratio & Argan group & D0 & $0.53 I \pm 0.086$ & $0.533 \pm 0.064$ & $>0.05$ \\
\hline \multirow[t]{3}{*}{$(U r / U f)$} & $\mathrm{N}=30$ & D60 & $0.594 \pm 0.059$ & $0.595 \pm 0.079$ & $>0.05$ \\
\hline & Olive group & D0 & $0.525 \pm 0.058$ & $0.525 \pm 0.085$ & $>0.05$ \\
\hline & $N=30$ & D60 & $0.55 I \pm 0.074$ & $0.607 \pm 0.075$ & 0.005 \\
\hline Resonance running time & Argan group & D0 & $103.70 \pm 17.24$ & $104.76 \pm 16.73$ & $>0.05$ \\
\hline \multirow[t]{3}{*}{$(\mathrm{AU})$} & $\mathrm{N}=30$ & D60 & $90.23 \pm 12.95$ & $87.09 \pm 14.33$ & $>0.05$ \\
\hline & Olive group & D0 & $102.1 \pm 15.26$ & $101.59 \pm 15.78$ & $>0.05$ \\
\hline & $N=30$ & D60 & $99.48 \pm 11.98$ & $91.29 \pm 14.82$ & 0.022 \\
\hline
\end{tabular}

Notes: All values are presented as mean \pm standard deviation. Mean group comparisons were conducted using Student's $t$-test for independent samples. $P<0.05$ was considered statistically significant.

Abbreviations: AU, arbitrary units; LF, left volar forearm; R2, Ua/Uf, gross-elasticity of the skin, including viscous deformation; R5, Ur/Ue, net elasticity of the skin without viscous deformation; R7, Ur/Uf, biological elasticity, ie, the ratio of immediate retraction to final distension; RF, right volar forearm.

In postmenopausal women, the decline in endogenous estrogen levels negatively affects skin elasticity, which causes a loss of skin collagen of up to $30 \%$ during the first 5 years after menopause ${ }^{3,18,19}$ or an average decline of $2.1 \%$ per postmenopausal year over 15 years. ${ }^{18}$ This also leads to the acceleration of degenerative changes in dermal elastic fibers, ${ }^{4}$ thus favoring the acceleration of skin aging.

This loss of collagen and elastic fibers may be the result of two mechanisms: the synthesis decrease and the degradation increase of collagen and elastic fibers. The decrease of collagen and elastic fibers synthesis would be due to the influence of hypoestrogenism on the synthesis of transforming growth factor-beta (TGF $\beta$ ), ${ }^{20}$ a cytokine involved in the activation of collagen and elastic fibers production via the signaling pathway. ${ }^{21}$ The increase of collagen and elastic fibers degradation would be due to the increase of reactive oxygen species (ROS) caused by the decrease of estrogen, representing the antioxidant of the human body, which induces an imbalance between the production of ROS and antioxidative defense observed at menopause, ${ }^{22}$ favoring the activation of matrix metalloproteinases (MMPs), ${ }^{23}$ a family of zinc-containing proteinases that have the ability to degrade collagen and elastic fibers. ${ }^{24}$

Estrogen treatment has improved skin elasticity by increasing collagen and elastic fibers synthesis, resulting from a decrease of TGF $\beta$, as well as by decreasing collagen and elastic fibers degradation, resulting from a decrease of MMPs.
This shows that estrogen treatment has a beneficial effect on reversing skin aging signs and maintaining skin elasticity in postmenopausal women, ${ }^{25}$ but its long-term use in the form of hormone replacement therapy triggers many pathophysiological events. ${ }^{9}$

The measurements of R-parameters have widely been used for determining biomechanical properties of human skin. The following R-parameters are known indicators of skin elasticity: ${ }^{26} \mathrm{R} 2$ is a measure of the gross elasticity of the skin, including viscous deformation, and is represented by the ratio of "the ability of redeformation of skin" to "final distension," ie, $\mathrm{Ua} / \mathrm{Uf},{ }^{16}$ or it is also known as the overall elasticity of the skin including creep and creep recovery ${ }^{27} \mathrm{R} 5$ is the so-called net elasticity of skin without viscous deformation. Elastic recovery is represented by the ratio of "immediate retraction" to "immediate distension," ie, $\mathrm{Ur} / \mathrm{Ue} .{ }^{16} \mathrm{R} 7$ is referred to as biological elasticity. This parameter relates elasticity with final distension. It is represented by the ratio of "immediate retraction" to "final distension," ie, Ur/Uf. ${ }^{16}$

In addition to the above measurements, skin elasticity measurements were also paralleled and confirmed by the RRT, measurements that represent the propagation time of a shear wave between two sensors placed on the skin surface; it has been shown that RRT is inversely related to skin elasticity. ${ }^{17}$

In this study, the results of the RF comparison showed an improvement in skin elasticity after 60 days of dietary 
argan oil consumption, which was demonstrated by a statistically significant increase of R-parameters (R2, R5, and R7), since the closer the values are to $1(100 \%)$, the more elastic the skin is. ${ }^{28}$ These increased R-parameters (R2, R5, and R7) were related to the increase of the content of collagen and elastic fibers of the skin, which agrees with the study by Ryu et al in which R2, R5, and R7 were negatively correlated to skin aging, ${ }^{29}$ characterized by the decrease of collagen and elastic fibers. In addition, these results of R-parameters were also confirmed by the statistically significant decrease of RRT, since the higher the measuring value, the less elastic the skin is, ${ }^{30}$ while no statistically significant improvement in skin elasticity was provided after the consumption of olive oil. As well, the comparison of the RF between the two groups at D60 showed a statistically significant improvement in skin elasticity in favor of the group consuming argan oil after the 60 days of consumption. In addition, in both groups, the results of the LF comparison showed a statistically significant improvement in skin elasticity after the 60 days of oils treatment. In the group consuming argan oil, these results of the LF reflected the more synergetic effect of the consumption and the application of argan oil; while in the group consuming olive oil, the results of the LF were due only to the effect of argan oil application, since the consumption of olive oil made no statistically significant improvement on RF. These results are due to the more abundant content of antioxidants in argan oil than that observed in olive oil. These antioxidants are mainly tocopherols (vitamin E), in addition to polyphenols, especially phenolic acid (ferulic acid). ${ }^{31-35}$ Vitamin E is a lipid-soluble nutrient that is derived from dietary intake of vegetable oils such as olive and argan oils. In addition to its antioxidant properties, ${ }^{36}$ vitamin $\mathrm{E}$ can act on skin elasticity by the downregulation of TGF $\beta,{ }^{37}$ resulting in a signal inducing fibroblasts to produce more collagen and elastic fibers necessary for the normal appearance and integrity of skin. Treatment with argan oil has increased the level of vitamin $\mathrm{E}$ in postmenopausal women's blood more than treatment with olive oil, ${ }^{38}$ which can explain the improvement of skin elasticity with argan oil treatment found in our study results. Ferulic acid is a phenolic compound found in argan and olive oil for some authors, ${ }^{33}$ and absent in olive oil composition for the others, ${ }^{34,35}$ known by its antioxidant properties. ${ }^{39}$ In Japan it has been approved as a food additive to prevent oxidation ${ }^{40}$ through its capacity to stay in the blood for longer than other antioxidants ${ }^{41}$ and favoring the neutralization of ROS.

The synergistic antioxidant effect of vitamin $\mathrm{E}$ and ferulic acid plays a key role in the maintenance of skin elasticity ${ }^{42,43}$ through the decline in collagen and elastic fibers degradation in response to MMP inhibition activity. That may help to protect postmenopausal women against the high levels of ROS implied in the acceleration of skin aging that takes place in age-related estrogen loss. ${ }^{22}$

So, vitamin E, synergistically with the ferulic acid present in argan oil, can replace the role of estrogen in the prevention of skin aging through the maintenance of skin elasticity in postmenopausal women.

This study has some limitations. One is the short duration of the intervention (60 days); the extension of this period was not possible, since most of the volunteers had other commitments. In addition to the inclusion of tocopherols and other argan oil chemical components (free fatty acid, phenol, and other compounds) are present in olive oil, with the exception of two essential sterols (schottenol and spinasterol), syringic acid, which have no known effects on skin elasticity, and phenolic acids (ferulic acid). ${ }^{31-35}$

Despite these limitations, this study shows a favorable trend of the efficiency of argan oil on skin elasticity of postmenopausal women and provides a good preliminary to a larger study. As well, no side effects were noted with the studied oil treatments during the study period. In addition, our population has probably benefited from the positive effect of argan oil in the prevention of disruptions or diseases related to menopause. ${ }^{13}$ Some of these effects (lipid and hormone profiles) are simultaneously being investigated by the other members of the research team.

\section{Conclusion}

The consumption and/or application of dietary and cosmetic argan oil, respectively, showed an ameliorative effect on skin elasticity of postmenopausal women after a 60-day period.

\section{Acknowledgments}

We are indebted to Hassan II Academy of Science and Agrotechnologies Association of Souss Massa Draa (Agrotech SMD) who funded our study; to Ibn Al Baytar association who was involved in the management of this study; to all the women who participated in the study; and also to Miss Rouchdi Mounia and Miss Zniber Meryem who accompanied these women during the intervention period.

\section{Author contributions}

Dr Bensouda had full access to all of the data in the study and takes responsibility for the integrity of the data and the accuracy of the data analysis. 
All authors contributed toward data analysis, drafting and revising the paper and agree to be accountable for all aspects of the work.

\section{Disclosure}

The authors report no conflicts of interest in this work.

\section{References}

1. Powell J, Soon CH. Physiology of the Skin. Surgery (Oxford). 2002; 20(6):ii-vi.

2. Bailey AJ, Etherington DJ. Metabolism of collagen and elastin. In: Florkin M, Neuberger A, Van Dienen LLM, editors. Comprehensive Biochemistry. New York: Elsevier Scientific; 1980:408-431.

3. Affinito P, Palomba S, Sorrentino C, et al. Effects of postmenopausal hypoestrogenism on skin collagen. Maturitas. 1999;33(3):239-247.

4. Bolognia JL, Braverman IM, Rousseau ME, Sarrel PM. Skin changes in menopause. Maturitas. 1989;11(4):295-304.

5. Calleja-Agius J, Brincat M, Borg M. Skin connective tissue and ageing. Best Pract Res Clin Obstet Gynaecol. 2013;27(5):727-740.

6. Sumino H, Ichikawa S, Kasama S, et al. Effects of raloxifene and hormone replacement therapy on forearm skin elasticity in postmenopausal women. Maturitas. 2009;62(1):53-57.

7. Punnonen R, Vaajalahti P, Teisala K. Local oestriol treatment improves the structure of elastic fibers in the skin of postmenopausal women. Ann Chir Gynaecol Suppl. 1987;202:39-41.

8. Sumino H, Ichikawa S, Abe M, Endo Y, Ishikawa O, Kurabayashi M. Effects of aging, menopause, and hormone replacement therapy on forearm skin elasticity in women. $J$ Am Geriatr Soc. 2004;52(6): 945-949.

9. Antoine C, Liebens F, Carly B, Pastijn A, Rozenberg S; Women's Health Initiative. Influence of HRT on prognostic factors for breast cancer: a systematic review after the Women's Health Initiative trial. Hum Reprod. 2004;19(3):741-756.

10. Berrougui H, Ettaib A, Herrera Gonzalez MD, Alvarez de Sotomayor M, Bennani-Kabchi N, Hmamouchi M. Hypolipidemic and hypocholesterolemic effect of argan oil (Argania spinosa L.) in Meriones shawi rats. J Ethnopharmacol. 2003;89(1):15-18.

11. Cherki M, Berrougui H, Drissi A, Adlouni A, Khalil A. Argan oil: which benefits on cardiovascular diseases? Pharmacol Res. 2006;54(1): $1-5$.

12. Bellahcen S, Mekhfi H, Ziyyat A, et al. Prevention of chemically induced diabetes mellitus in experimental animals by virgin argan oil. Phytother Res. 2012;26(2):180-185.

13. Azizi F, Ainy E. Coronary heart disease risk factors and menopause: a study in 1980 Tehranian women, the Tehran Lipid and Glucose Study. Climactric. 2003;6(4):330-336.

14. Bellakhdar J. La pharmacopée marocaine traditionnelle [Traditional Moroccan pharmacopoeia]. Ibis Press: Paris; 1997.

15. Derouiche A, Cherki M, Drissi A, et al. Nutritional intervention study with argan oil in man: Effects on lipids and apolipoproteins. Ann Nutr Metab. 2005;49(3):196-201.

16. Dobrev H. Use of Cutometer to assess epidermal hydration. Skin Res Technol. 2000;6(4):239-244.

17. Paye M, Mac-Mary S, Elkhyat A, Tarrit C, Mermet P, Humbert PH. Use of the Reviscometer for measuring cosmetics-induced skin surface effects. Skin Res Technol. 2007;13(4):343-349.

18. Brincat M, Kabalan S, Studd JW, Moniz CF, de Trafford J, Montgomery J. A study of the decrease of skin collagen content, skin thickness, and bone mass in the postmenopausal woman. Obstet Gynecol. 1987;70(6):840-845.

19. Brincat MP. Hormone replacement therapy and the skin. Maturitas. 2000;35(2):107-117.
20. Son ED, Lee JY, Lee S, et al. Topical application of 17beta-estradiol increases extracellular matrix protein synthesis by stimulating tgf-Beta signaling in aged human skin in vivo. J Invest Dermatol. 2005;124(6): 1149-1161.

21. Duncan MR, Frazier KS, Abramson S, et al. Connective tissue growth factor mediates transforming growth factor b-induced collagen synthesis: downregulation by cAMP. FASEB J. 1999;13(13):1774-1786.

22. Sánchez-Rodríguez MA, Zacarías-Flores M, Arronte-Rosales A, Correa-Muñoz E, Mendoza-Núñez VM. Menopause as risk factor for oxidative stress. Menopause. 2012;19(3):361-367.

23. Calleja-Agius J, Brincat M, Borg M. Skin connective tissue and ageing. Best Pract Res Clin Obstet Gynaecol. 2013;27(5):727-740.

24. Kähäri VM, Saarialho-Kere U. Matrix metalloproteinases and their inhibitors in tumour growth and invasion. Ann Med. 1999;31(1):34-45.

25. Sator PG, Sator MO, Schmidt JB, et al. A prospective, randomized, double-blind, placebo controlled study on the influence of a hormone replacement therapy on skin aging in postmenopausal women. Climacteric. 2007;10(4):320-334.

26. Cho S, Lee S, Lee MJ, et al. Dietary Aloe Vera supplementation improves facial wrinkles and elasticity and it increases the type I procollagen gene expression in human skin in vivo. Ann Dermatol. 2009;21(1): 6-11.

27. Ryu HS, Joo YH, Kim SO, Park KC, Youn SW. Influence of age and regional differences on skin elasticity as measured by the Cutometer. Skin Res Technol. 2008;14(3):354-358.

28. Akhtar N, Waqas MK, Ahmed M, et al. Effect of cream formulation of fenugreek seed extract on some mechanical parameters of human skin. Trop J Pharm Res. 2010;9(4):329-337.

29. Ryu HS, Joo YH, Kim SO, Park KC, Youn SW. Influence of age and regional differences on skin elasticity as measured by the Cutometer. Skin Res Technol. 2008;14(3):354-358.

30. Hermanns-Lê T, Jonlet F, Scheen A, Piérard GE. Age- and body mass index-related changes in cutaneous shear wave velocity. Exp Gerontol. 2001;36(2):363-372.

31. Charrouf Z, Guillaume D. Argan oil: Occurrence, composition and impact on human health. Eur J Lipid Sci Technol. 2008;110(7): 632-636.

32. Ollivier D, Artaud J, Pinatel C, Durbec JP, Guérère M. Triacylglycerol and fatty acid compositions of French virgin olive oils. Characterization by chemometrics. J Agric Food Chem. 2003;51(19):5723-5731.

33. Khallouki F, Younos C, Soulimani R, et al. Consumption of argan oil (Morocco) with its unique profile of fatty acids, tocopherols, squalene, sterols and phenolic compounds should confer valuable cancer chemopreventive effects. Eur J Cancer Prev. 2003;12(1):67-75.

34. Tuberoso CIG, Kowalczyk A, Sarritzu E, Cabras P. Determination of antioxidant compounds and antioxidant activity in commercial oilseeds for food use. Food Chem. 2007;103(4):1494-1501.

35. Becerra-Herrera M, Sánchez-Astudillo M, Beltrán R, Sayago A. Determination of phenolic compounds in olive oil: New method based on liquid-liquid micro extraction and ultra high performance liquid chromatography-triple-quadrupole mass spectrometry. LWT-Food Sci Technol. 2014;57(1):49-57.

36. Traber MG, Atkinson J. Vitamin E, antioxidant and nothing more. Free Radic Biol Med. 2007;43(1):4-15.

37. Parola M, Muraca R, Dianzani I, et al. Vitamin E dietary supplementation inhibits transforming growth factor beta 1 gene expression in the rat liver. FEBS Lett. 1992;308(3):267-270.

38. El Monfalouti H, Charrouf Z, El Hamdouchi A, et al. Argan oil and postmenopausal Moroccan women: impact on the vitamin E profile. Nat Prod Commun. 2013;8(1):55-57.

39. Kikuzaki H, Hisamoto M, Hirose K, Akiyama K, Taniguchi H. Antioxidant properties of ferulic acid and its related compounds. $J$ Agric Food Chem. 2002;50(7):2161-2168.

40. Graf E. Antioxidant potential of ferulic acid. Free Radic Biol Med. 1992;13(4):435-448. 
41. Itagaki S, Kurokawa T, Nakata C, et al. In vitro and in vivo antioxidant properties of ferulic acid: A comparative study with other natural oxidation inhibitors. Food Chem. 2009;114(2):466-471.

42. Manosroi A, Chutoprapat R, Abe M, Manosroi W, Manosroi J. Anti-aging efficacy of topical formulations containing niosomes entrapped with rice bran bioactive compounds. Pharm Biol. 2012;50(2):208-224.
43. Masaki H. Role of antioxidants in the skin: Anti-aging effects. J Dermatol Sci. 2010;58(2):85-90.

\section{Publish your work in this journal}

Clinical Interventions in Aging is an international, peer-reviewed journal focusing on evidence-based reports on the value or lack thereof of treatments intended to prevent or delay the onset of maladaptive correlates of aging in human beings. This journal is indexed on PubMed Central, MedLine,

\section{Dovepress}

CAS, Scopus and the Elsevier Bibliographic databases. The manuscript management system is completely online and includes a very quick and fair peer-review system, which is all easy to use. Visit http://www.dovepress. $\mathrm{com} /$ testimonials.php to read real quotes from published authors. 